\section{A common polymorphism in SNCA is associated with accelerated motor decline in GBA-Parkinson's disease}

A growing number of genetic susceptibility factors have been identified for Parkinson's disease (PD). The combination of inherited risk variants is likely to affect not only risk of developing PD but also its clinical course. Variants in the GBA gene are particularly common, being found in approximately 5\% to $10 \%$ of patients, and they lead to more rapid disease progression. ${ }^{1}$ However, the effect of concomitant genetic risk factors on disease course in GBA-PD is not known.

The aggregation of $\alpha$-synuclein, encoded by the SNCA gene, is central to the pathogenesis of PD. The SNCA rs356219 A/G polymorphism alters the risk of developing $\mathrm{PD}$, with homozygotes for guanine $(\mathrm{G} / \mathrm{G})$ having an increased risk compared with carriers of an adenine (G/A or $\mathrm{A} / \mathrm{A})$ at this locus. ${ }^{2}$ The relationship between glucocerebrosidase (the enzyme encoded by the $G B A$ gene) and $\alpha$-synuclein is complex. These proteins have been shown to interact directly in vitro, as well as to influence the intracellular levels and processing of each other, potentially in a bidirectional feedback loop. ${ }^{34}$ Interestingly, a recent genome-wide association study found that the presence of this SNCA polymorphism was associated with an increased likelihood of developing PD in GBA mutation carriers. ${ }^{5}$ We therefore hypothesised that the presence of the SNCA rs356219 polymorphism would accelerate the clinical course of $G B A$ variant-associated PD. Here, we report on the effect of this SNCA polymorphism on clinical outcomes within the GBA-PD population.

Longitudinal data from $G B A$-variant carriers were analysed from the communitybased 'Cambridgeshire Incidence of Parkinson's disease from General Practice to Neurologist' (CamPaIGN) cohort $(n=142){ }^{6}$ This study was approved by the local ethics committee and written informed consent was obtained from all subjects. Newly diagnosed patients were followed up with assessments every 2 years for up to 18 years. Time to development of dementia (defined as Mini-Mental State Examination (MMSE) score of 24 or less, with fulfilment of Diagnostic and Statistical Manual of Mental Disorders IV criteria), progression to postural instability (Hoehn and Yahr stage three (HY3)) and death were determined.

Sequencing of the GBA gene was carried out in 114 patients in the CamPaIGN cohort, as described here. ${ }^{7}$ A further 16 patients underwent targeted genetic screening for common $G B A$ variants using the Illumina Multi-Ethnic Genotyping Array (MEGA) chip. Genetic analysis of the SNCA rs356219 polymorphism had also been previously performed in 124 patients from the cohort. ${ }^{2} G B A$ variants were identified in 20 patients, which were included in survival analyses. Of these, 7 carried the high-risk (G/G) SNCA genotype, with 13 carrying low-risk (G/A or $\mathrm{A} / \mathrm{A})$ genotypes. There were no differences in age at diagnosis nor in baseline assessments including total and part III Unified Parkinson's Disease Rating Scale scores, Hoehn and Yahr scores, MMSE scores or levodopa equivalent doses between these SNCA genotype groups. All carriers of the $\mathrm{G} / \mathrm{G}$ genotype were male, compared with $53.8 \%$ in the low-risk group $(p=0.03)$ (online supplementary table 1).

Survival analyses for the aforementioned outcome measures were performed, with statistical significance determined using log-rank tests. P-values were adjusted for multiple comparisons using the Bonferroni method. Among carriers of GBA variants, there were no differences between the high-risk and low-risk SNCA groups for time to dementia $(p=0.29$, adjusted $\mathrm{p}=0.86)$ or death $(\mathrm{p}=0.43$, adjusted $\mathrm{p}=1.28$ ) (figure 1A,B). Progression to $\mathrm{HY} 3$, however, was significantly faster in the $\mathrm{G} / \mathrm{G} S N C A$ group $(\mathrm{p}=0.02$, adjusted $\mathrm{p}=0.07$ ), with mean time to development of postural instability 2.0 years $(95 \% \mathrm{CI}$ 1.3 to 2.7$)$ compared with 4.9 years $(95 \%$ CI 2.5 to 7.3 ) in A carriers (figure 1C). All
G/G carriers reached HY3 within 3 years of diagnosis.

To account for potential confounders, time to HY3 was also compared using a Cox regression model controlling for sex and age at diagnosis. The G/G SNCA genotype was associated with a HR for progression to HY3 of 3.8 (95\% CI 0.9 to 16.1 ), $\mathrm{p}=0.07$, adjusted $\mathrm{p}=0.21$ ) when controlling for these confounders. Because all the SNCA $\mathrm{G} / \mathrm{G}$ carriers were male, we also performed a survival analysis in which only male subjects were included, to further control for sex as a potential confounder. An increased risk of progression to $\mathrm{HY} 3$ was again observed in $\mathrm{G} / \mathrm{G}$ carriers in comparison to A carriers $(\mathrm{p}=0.036$, adjusted $\mathrm{p}=0.11$; online supplementary figure 1). SNCA genotype had no effect on progression to $\mathrm{HY} 3$ in non-carriers of $G B A$ variants $(\mathrm{n}=85)$ in the CamPaIGN cohort $(\mathrm{p}=0.88$, adjusted $\mathrm{p}=2.65$; online supplementary figure 2 ).

This cohort contained four patients with the non-coding c. 762-18 $\mathrm{T}>\mathrm{A}$ variant (online supplementary table 2 ). While this has been reported to be a potential risk factor for $\mathrm{PD},{ }^{8}$ its importance is not clear. We therefore also performed analysis after exclusion of these patients and found that progression to $\mathrm{HY} 3$ was greater in the $\mathrm{G} / \mathrm{G}$ carrier group, with a HR of $5.3(95 \% \mathrm{CI}$ 1.1 to $26.2 ; \mathrm{p}=0.041$, adjusted $\mathrm{p}=0.12$ ).

To our knowledge, no previous studies have investigated the influence of concomitant genetic risk factors on the progression of GBA-PD. Here we report that the SNCA rs356219 polymorphism significantly affects motor progression in GBA-PD, with the $G / G$ genotype associated with a particularly aggressive disease course. This effect was not observed in patients with PD without $G B A$ variants, suggesting that it was relatively specific to $G B A-P D$.

This new study therefore raises the interesting possibility that $G B A$ variants and the G/G SNCA rs356219 polymorphism act synergistically to accelerate pathology and clinical progression in PD. GBA mutations are thought to increase the risk of PD predominantly through perturbations in the lysosome-autophagy system-a system important in $\alpha$-synuclein clearance.
A

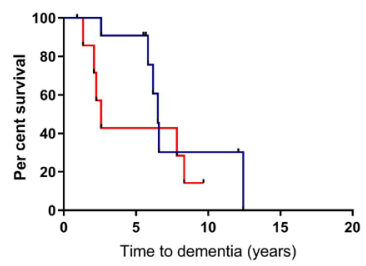

B

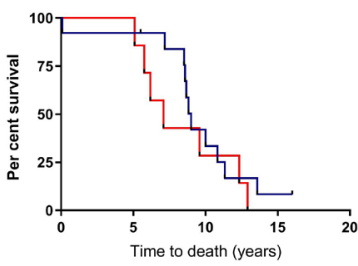

C

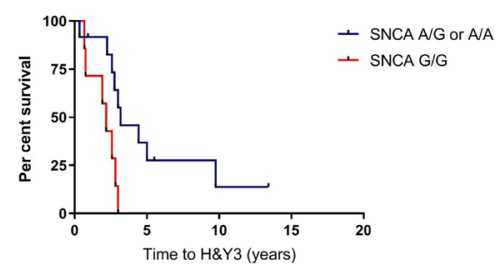

Figure 1 Survival analyses in GBA-PD patients comparing those with high-risk and low-risk variants in the SNCA rs356219 polymorphism. Kaplan-Meier curves for time to (A) dementia, $(B)$ death and (C) postural instability. A, adenine, G, guanine; H\&Y3, Hoehn and Yahr stage three; PD, Parkinson's disease. 
Furthermore, glucocerebrosidase and $\alpha$-synuclein have been shown to interact directly in vitro and to coexist in Lewy bodies of patients with PD, with a greater proportion of Lewy bodies containing glucocerebrosidase in patients with GBA mutations compared with those in patients with PD not carrying a $G B A$ variant. ${ }^{49}$ It has been speculated that mutant glucocerebrosidase potentiates the aggregation of $\alpha$-synuclein, and it is therefore feasible that SNCA variants such as the rs356219 polymorphism alters the degree to which $G B A$ variants predispose to PD pathology and disease progression. It has also been suggested that $\alpha$-synuclein impedes the transit of glucocerebrosidase from the endoplasmic reticulum to the lysosome, further supporting the idea that the relationship between these two proteins is directly important in the pathogenesis of GBA-PD. ${ }^{3}$ The proposed synergistic interaction between variants in the GBA gene and SNCA gene therefore could also potentially be explained by differences in the degree to which $\alpha$-synuclein impairs normal glucocerebrosidase processing associated with specific $S N C A$ variants.

We acknowledge that our sample size is small, but these preliminary observations raise the possibility that $G B A$ variants and the G/G SNCA rs356219 polymorphism synergistically alter motor progression in PD. In this study we have considered the time from diagnosis to the development of important clinical milestones in PD. The time to diagnosis may vary between patients, so it should be recognised that this does not necessarily reflect disease duration. Time from disease onset to the development of clinical milestones may be more representative of disease duration, but onset is very difficult to ascertain accurately, given that many patients experience prodromal symptoms that are not initially attributed to PD.

There is a degree of genotype-phenotype correlation in GBA-PD, with severe mutations accelerating disease course to a greater extent than less severe variants. ${ }^{10}$ However, because our sample size was small, it was not possible to stratify the patients with $G B A-P D$ into those with non-severe and severe GBA variants, so our GBA-PD population was genetically heterogeneous (online supplementary table 2). Mean age at diagnosis was approximately 5 years later in $\mathrm{G} / \mathrm{G}$ carriers which may potentially have contributed to their accelerated disease course. However, there were no differences in time to dementia or death, and when age at diagnosis was accounted for in the Cox regression model, the accelerated progression to HY3 persisted. Another limitation of our study is that all subjects in the G/G group were male, and though this was accounted for in the Cox regression model and by performing the additional maleonly analysis, it would be important to investigate the relationship between these two genetic risk factors in a large cohort, to reduce the effect of such confounders and to allow for the stratification of $\mathrm{G} / \mathrm{G}$ carriers into those with severe and those with mild $G B A$ variants.

\section{Thomas B Stoker ${ }^{10},{ }^{1,2}$ Marta Camacho, ${ }^{1}$ Sophie Winder-Rhodes, ${ }^{1}$ Ganqiang Liu, ${ }^{3,4,5}$ Clemens R Scherzer, ${ }^{4,5}$ Thomas Foltynie $\odot{ }^{6}{ }^{6}$ Roger A Barker, ${ }^{1,2}$ Caroline H Williams-Gray ${ }^{1}$}

${ }^{1}$ John van Geest Centre for Brain Repair, Department of Clinical Neurosciences, University of Cambridge, Cambridge, UK

${ }^{2}$ Wellcome Trust - Medical Research Council Stem Cell Institute, University of Cambridge, Cambridge, UK

${ }^{3}$ School of Medicine, Sun Yat-Sen University,

Guangzhou, Guangdong, China

${ }^{4}$ Advanced Center for Parkinson's Disease Research, Harvard Medical School, Brigham and Women's Hospital, Boston, Massachusetts, USA

${ }^{5}$ Precision Neurology Program, Harvard Medical School, Brigham and Women's Hospital, Boston, Massachusetts, USA

${ }^{6}$ Department of Clinical and Movement Neurosciences, UCL Institute of Neurology, London, UK

Correspondence to Dr Thomas B Stoker, John van Geest Centre for Brain Repair, Department of Clinical Neurosciences, University of Cambridge, Cambridge, Cambridgeshire CB2 OPY, UK; tbs26@cam.ac.uk

\section{Twitter Thomas Foltynie @foltynie}

Contributors TBS wrote the manuscript. TBS, MC, $S W-R, G L$ and TF performed the research. TF, CRS, $R A B$ and $C H W-G$ developed the original concepts and provided critical review of the manuscript.

Funding The CamPaIGN study has received financial support from the Wellcome Trust, the Medical Research Council, Parkinson's UK and the Patrick Berthoud Trust. CHW-G is supported by a Research Councils UK/UK Research and Innovation Fellowship awarded by the Medical Research Council (MR/R007446/1). RAB is supported by the Wellcome Trust Stem Cell Institute (Cambridge 203151/Z/16/Z). RAB is a National Institute for Health Research (NIHR) Senior Investigator (NFSI-0616-10011). TBS received financial support from the Cure Parkinson's Trust. The study is also supported by the NIHR Cambridge Biomedical Research Centre Dementia and Neurodegeneration Theme (reference number 146281). CRS' work is supported in part by National Institutes of Health grants R01AG057331, U01NS100603, R01AG057331, and the American Parkinson Disease Association. Illumina MEGA Chip genotyping was made possible by a philanthropic investment from Dooley LLC (to Brigham \& Women's Hospital and (RS).

Competing interests None declared.

Patient consent for publication Not required.

Provenance and peer review Not commissioned; externally peer reviewed.

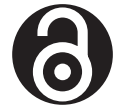

OPEN ACCESS
Open access This is an open access article distributed in accordance with the Creative Commons Attribution 4.0 Unported (CC BY 4.0) license, which permits others to copy, redistribute, remix, transform and build upon this work for any purpose, provided the original work is properly cited, a link to the licence is given, and indication of whether changes were made. See: https://creativecommons.org/licenses/by/ $4.0 \%$.

(C) Author(s) (or their employer(s)) 2020. Re-use permitted under CC BY. Published by BMJ.

- Additional material is published online only. To view please visit the journal online (http://dx.doi.org/ 10.1136/jnnp-2019-322210)

\section{Check for updates}

To cite Stoker TB, Camacho M, Winder-Rhodes S, et al. J Neurol Neurosurg Psychiatry 2020;91:673-674.

Received 9 October 2019

Revised 18 February 2020

Accepted 9 March 2020

Published Online First 2 April 2020

J Neurol Neurosurg Psychiatry 2020;91:673-674. doi:10.1136/jnnp-2019-322210

\section{ORCID iDs}

Thomas B Stoker http://orcid.org/0000-0001-51867630

Thomas Foltynie http://orcid.org/0000-0003-07521813

\section{REFERENCES}

1 Cilia R, Tunesi S, Marotta G, et al. Survival and dementia in GBA-associated Parkinson's disease: the mutation matters. Ann Neurol 2016;80:662-73.

2 Goris A, Williams-Gray CH, Clark GR, et al. Tau and alpha-synuclein in susceptibility to, and dementia in, Parkinson's disease. Ann Neurol 2007;62:145-53.

3 Mazzulli JR, Xu Y-H, Sun Y, et al. Gaucher disease glucocerebrosidase and $\alpha$-synuclein form a bidirectional pathogenic loop in synucleinopathies. Cell 2011;146:37-52.

4 Yap TL, Velayati A, Sidransky E, et al. Membranebound $\alpha$-synuclein interacts with glucocerebrosidase and inhibits enzyme activity. Mol Genet Metab 2013;108:56-64.

5 Blauwendraat C, Reed X, Krohn L, et al. Genetic modifiers of risk and age at onset in GBA associated Parkinson's disease and Lewy body dementia. Brain 2020;143:234-48.

6 Williams-Gray CH, Mason SL, Evans JR, et al. The campaign study of Parkinson's disease: 10-year outlook in an incident population-based cohort. J Neurol Neurosurg Psychiatry 2013;84:1258-64.

7 Winder-Rhodes SE, Evans JR, Ban M, et al. Glucocerebrosidase mutations influence the natural history of Parkinson's disease in a community-based incident cohort. Brain 2013;136:392-9.

8 Moors TE, Paciotti S, Ingrassia A, et al. Characterization of brain lysosomal activities in GBA-Related and sporadic Parkinson's disease and dementia with Lewy bodies. Mol Neurobiol 2019;56:1344-55.

9 Goker-Alpan 0, Stubblefield BK, Giasson BI, et al. Glucocerebrosidase is present in $\alpha$-synuclein inclusions in Lewy body disorders. Acta Neuropathol 2010;120:641-9.

10 Liu G, Boot B, Locascio JJ, et al. Specifically neuropathic Gaucher's mutations accelerate cognitive decline in Parkinson's. Ann Neurol 2016;80:674-85. 